\title{
High spatial resolution studies of phase transitions within organic aperiodic crystals
}

\author{
Céline Mariette $\odot,{ }^{1, *}$ Laurent Guérin,,${ }^{1} \dagger$ Philippe Rabiller, ${ }^{1}$ Christophe Odin, ${ }^{1}$ Mariana Verezhak $\odot,{ }^{1,2}$ Alexei Bosak, ${ }^{3}$ \\ Philippe Bourges, ${ }^{4}$ Claude Ecolivet, ${ }^{1}$ and Bertrand Toudic ${ }^{1}{ }^{1, \ddagger}$ \\ ${ }^{1}$ Univ. Rennes, CNRS, IPR (Institut de Physique de Rennes) - UMR 6251, F-35000 Rennes, France \\ ${ }^{2}$ Paul Scherrer Institut, Forschungsstrasse 111, 5232 Villigen, Switzerland \\ ${ }^{3}$ European Synchrotron Radiation Facility, BP 220, 38043 Grenoble Cedex, France \\ ${ }^{4}$ Université Paris-Saclay, CNRS, CEA, Laboratoire Léon Brillouin, 91191 Gif-sur-Yvette, France
}

(Received 20 December 2019; revised manuscript received 28 February 2020; accepted 7 April 2020; published 11 May 2020)

\begin{abstract}
The understanding of the symmetry breakings within crystals that are aperiodic by construction is actually very limited. Quasicrystals and incommensurate composite crystals may potentially allow such studies. We focus on the phase transitions of the aperiodic $n$-nonadecane/urea which recovers a translational symmetry within a four-dimensional space at room temperature. High-resolution neutron and synchrotron studies are reported as a function of the temperature on this organic crystal which presents an exceptional mosaicity. They reveal the richness of such approach, showing the appearance of very long wavelength supplementary intermodulations. This work generalizes the Landau theory to incommensurate composite crystals.
\end{abstract}

DOI: 10.1103/PhysRevB.101.184107

\section{INTRODUCTION}

Phase transitions and critical phenomena in matter have been fundamental fields of research for the past century. Huge progress was made in formalism with the Landau mean-field theory and, later on, with the renormalization-group theory $[1,2]$. In parallel, the field extends to crystals which are not periodic in the three-dimensional space. Simultaneously, a huge breakthrough came from the experimental side. Synchrotron radiations offer high flux with low divergence, which allowed the development of highly selective techniques leading to exceptional resolution in reciprocal space. However, in order to reap the benefit of these ultrahigh spatial resolutions, some conditions are required for the studied crystal itself. There, it appears that organic crystals may present such a perfect mosaicity that it is not even measurable on the best highresolution spectrometers. This opens the possibility to study deeper symmetry-breaking properties around phase transitions such as critical phenomena or within aperiodic crystals. The new definition of the crystal by the International Union of Crystallography includes the materials that present longrange order without translational symmetry. The periodicity of such crystals is recovered in higher-dimensional spaces, called crystallographic superspaces [3-6], that are characterized by more than three independent basis vectors. The quasiperiodic crystals are usually divided into three families: the incommensurately modulated ones, the incommensurate composite crystals, and the quasicrystals. Much work has been dedicated to these three families, focusing on their structures, their dynamics, and other physical properties [7-12]. Concerning

\footnotetext{
*celine.mariette@univ-rennes1.fr

†laurent.guerin@univ-rennes1.fr

‡bertrand.toudic@univ-rennes1.fr
}

structural instabilities in these materials, the incommensurate modulated crystals have been extensively and definitively successfully explained [6]. Indeed, the incommensurate phase is the result of a phase transition from a three-dimensional periodic space to $(3+1)$ or $(3+2)$ dimensions where their periodicity is recovered. The superspace group describing these incommensurate phases is directly related to the periodic space group of the high-symmetry phase $[6,7,13,14]$. Quasicrystals and incommensurate composite crystals do not present any high-symmetry periodic phase, which makes their studies of course much more difficult. Phase transitions within quasicrystals have not really been analyzed in the literature. More works have been dedicated to incommensurate composite crystals [7,15-19]. A peculiar case corresponds to host/guest intergrowth nanotubular structures, which have a sole incommensurate direction, called $\boldsymbol{c}$ here. There, periodicity is recovered in a four-dimensional space and a fourdimensional superspace description gives the positions of the complete set of Bragg peaks [3,4,7]: $\boldsymbol{Q}_{h k l m}=h \boldsymbol{a}^{*}+k \boldsymbol{b}^{*}+$ $l \boldsymbol{c}_{h}^{*}+m \boldsymbol{c}_{g}^{*}$, where $\boldsymbol{a}^{*}, \boldsymbol{b}^{*}, \boldsymbol{c}_{h}^{*}$, and $\boldsymbol{c}_{g}^{*}$ are the conventional reciprocal unit-cell vectors, and $\boldsymbol{c}_{h}$ and $\boldsymbol{c}_{g}$ refer, respectively, to the host and the guest parameters along the aperiodic direction. Four indices are needed to describe four different types of structure Bragg peaks $(h k l m$ ): a convenient but simplistic labeling is that $(h k 00),(h k l 0),(h k 0 \mathrm{~m})$, and ( $h k l \mathrm{~m}$ ), with $l$ and $m$ different from zero, are the common, host, guest, and satellite Bragg peaks, respectively. Within this family of aperiodic intergrowth nanotubular structures, the organic guest/host $n$-alkane/urea compounds constitute a prototype family [7,20-39]. In almost all of the $n$-alkane/urea inclusion compounds, a four-dimensional hexagonal superspace group $P 6_{1} 22(00 \gamma)$ is reported in the literature at room temperature. The parameters for the host urea subsystem are then $a_{\text {hex }}=b_{\text {hex }}=8.22 \AA$, and the pitch of the urea helix is $c_{\text {host }}=c_{h}=11.02 \AA$ [27]. In the case of hydrogenated 
(linear) n-alkane/urea, an empirical experimental estimation of the guest periodicity $c_{g}$ was reported by Lenné et al. [21]: $c_{g}=[1.277(\mathrm{n}-1)+3.48] \AA$, where $\mathrm{n}$ is the number of carbon atoms in the alkane molecule. In most cases, the ratio of the host and guest parameter is irrational, defining a misfit parameter $\gamma=\left(c_{h} / c_{g}\right)=\left(c_{g}^{*} / c_{h}^{*}\right)$. These compounds are then aperiodic by construction. They present structural phase transitions which have been studied for many years. However, in earlier studies, aperiodicity was not considered as important for the single-phase transition detected at that time. Consequently, theoretical works limited their description to the common periodic $(\boldsymbol{a}, \boldsymbol{b})$ plane where a ferroelastic shear occurs [22-24,26,40]. Somehow, this corresponds to the simplistic description of a two-dimensional problem and not a three, and a fortiori not a four-dimensional one. Due to the lack of resolution, they wrongly observed the appearance of superstructure Bragg peaks within the basal $\left(\boldsymbol{a}^{*}, \boldsymbol{b}^{*}\right)$ plane and thus they concluded it was an antiferrodistorsion of the host urea sublattice and a herringbone ordering of the guest alkane molecules [24-26]. Considering the aperiodic feature of the crystal, we reported experimental results and theoretical interpretations within crystallographic superspaces in total contradiction with this established interpretation. Much of our work was dedicated to $n$-nonadecane/urea [31,33,35]. Reference [31] concerns experimental data performed at atmospheric pressure within the so-called phases II and III of $n$ nonadecane/urea, whereas Ref. [33] reports experimental data under pressure at $0.5 \mathrm{GPa}$ in a different phase, called phase IV. In this paper, the superspace groups of these different phases are given. According to adiabatic calorimetric measurements, several phase transitions were reported in these crystals, with the transition temperatures presenting isotopic effects [41]. These transition temperatures are $T_{c 1}=158.8 \mathrm{~K}$ and $T_{c 2}=147 \mathrm{~K}$ for the fully hydrogenated $\mathrm{C}_{19} \mathrm{H}_{40} / \mathrm{CO}\left(\mathrm{NH}_{2}\right)_{2}$ compound and at $T_{c 1}^{D}=149.4 \mathrm{~K}$ and $T_{c 2}^{D}=127.8 \mathrm{~K}$ for the fully deuterated $C_{19} D_{40} / C O\left(N D_{2}\right)_{2}$. Very recently, some authors questioned this work $[42,43]$. They argue in favor of a single ferroelastic phase transition described in the $(\boldsymbol{a}, \boldsymbol{b})$ plane $[26,44]$. We strongly contested this work $[45,46]$. Here we present high-resolution synchrotron data which prove the originality of the phase transitions when described by taking into account their aperiodic feature by construction.

\section{SYMMETRY-BREAKING-INDUCED DOMAINS}

Symmetry breaking was extensively studied many years ago considering the symmetry-induced domains. At that time, a single-phase transition was considered in $n$-alkane/urea compounds at a transition temperature $T_{c}$, that we will call $T_{c 1}$ here. The phase transition was observed from hexagonal to orthorhombic with the appearance of a splitting of the basal Bragg peaks. They correspond to differently oriented domains. These domains result from the loss of the sixfold screw axis along the channel direction. The two sets of three domains (1, 2, and 3) further rotate by a small angle of the order of \pm 1 degree, as shown by Forst et al. [24], and that is explained in terms of energetic arrangements. Weber [40] observed the existence of supplementary smaller domains with a double rotational angle. Our neutron-scattering experiments were performed in the Laboratoire Léon Brillouin at the Orphée reactor (Saclay, France). The diffraction data were collected on the triple-axis spectrometer $1 \mathrm{~T}$ installed on a thermal neutron source. The incident wave vector was $k_{i}=$ $2.662 \AA^{-1}$. A standard setup was used with no collimations and a Pyrolytic Graphite filter was installed on $k_{f}$ to remove the higher-order harmonics. The retained scattering plane was the periodic $\left(\boldsymbol{a}^{*}, \boldsymbol{b}^{*}\right)$ one. With this study of fully deuterated $n$-nonadecane/urea on a much bigger crystal than used for an $\mathrm{x}$ ray, we confirm the above experimental result also with the same proportion of these domains (1, 2, and 3). The supplementary small domains are denoted by an asterisk in Fig. 1. In the following, we will focus on the diffraction signature of $n$-nonadeacane/urea along the direction perpendicular to the $\left(\boldsymbol{a}^{*}, \boldsymbol{b}^{*}\right)$ plane using cold neutron scattering and x-ray synchrotron diffraction. In both cases, the spatial resolution in the reciprocal space allows the selection of one single domain, the so-called domain $1+$ in Fig. 1.

\section{THE SEQUENCE OF PHASES}

Several articles have already been dedicated to the measurements along the reciprocal channel axis of $n$ nonadecane/urea [28,29,31,33,35,47]. The first papers proved the aperiodic feature of $n$-nonadecane/urea, showing the existence of the structure Bragg peaks appearing at combinatory positions, that is, Bragg peaks with the indices $(h, k, l, m)$, with $l$ and $m$ simultaneously different from zero [28,29,47]. This was a proof of the reciprocal intermodulation of the host (urea) and the guest (n-nonadecane) sublattices. The other papers $[31,33,35]$ focused on the phase transitions that appear in this crystal as a function of the temperature. In order to drastically reduce the incoherent neutron scattering of the hydrogen atoms, fully deuterated crystals actually have to be used for the neutron-scattering studies. Figure 2 reports the diffraction profile measured using neutron scattering on the fully deuterated crystal along the $c^{*}$ direction, perpendicular to the $\left(\boldsymbol{a}^{*}, \boldsymbol{b}^{*}\right)$ plane. Neutron-scattering experiments were performed in the Laboratoire Léon Brillouin at the Orphée reactor (Saclay, France). The diffraction data were collected on the triple-axis spectrometer $4 \mathrm{~F}$ installed on a cold neutron source. The incident wave vector was $k_{i}=2.662 \AA^{-1}$. Since the energy resolution is of the order of $100 \mu \mathrm{eV}$, it means that phonons are not integrated within the presented elastic measurements. The retained scattering plane was the aperiodic $\left(3 \boldsymbol{a}^{*}+2 \boldsymbol{b}^{*}, \boldsymbol{c}^{*}\right)$ plane, according to the domain $1+$ orthorhombic notation. The scan ranges from $l=-1.5$ to 0.5 , which is around the host reciprocal positions $(l=-1)$ and around the common Bragg peak $(l=0)$. In the case of the fully deuterated $n$-nonadecane/urea, the first low symmetry phase, called phase II, spreads between $T_{c 1}^{D}=149.4 \mathrm{~K}$ and $T_{c 2}^{D}=127.8 \mathrm{~K}$. The systematic absence of the common and host superstructure Bragg peaks (defined by $h+k$ odd in the orthorhombic low-symmetry domain $1+$ ) in this phase has been discussed extensively in previous articles [33]. A supplementary intermodulation of the crystal appears within this phase II. It is associated to the appearance of strong Bragg peaks, very slightly away from the common reciprocal position, as shown in Fig. 2 (top). A similar feature appears around the host reciprocal position. These Bragg peaks are located at $\pm \delta c_{h}^{*}$ apart from these two reciprocal positions. 

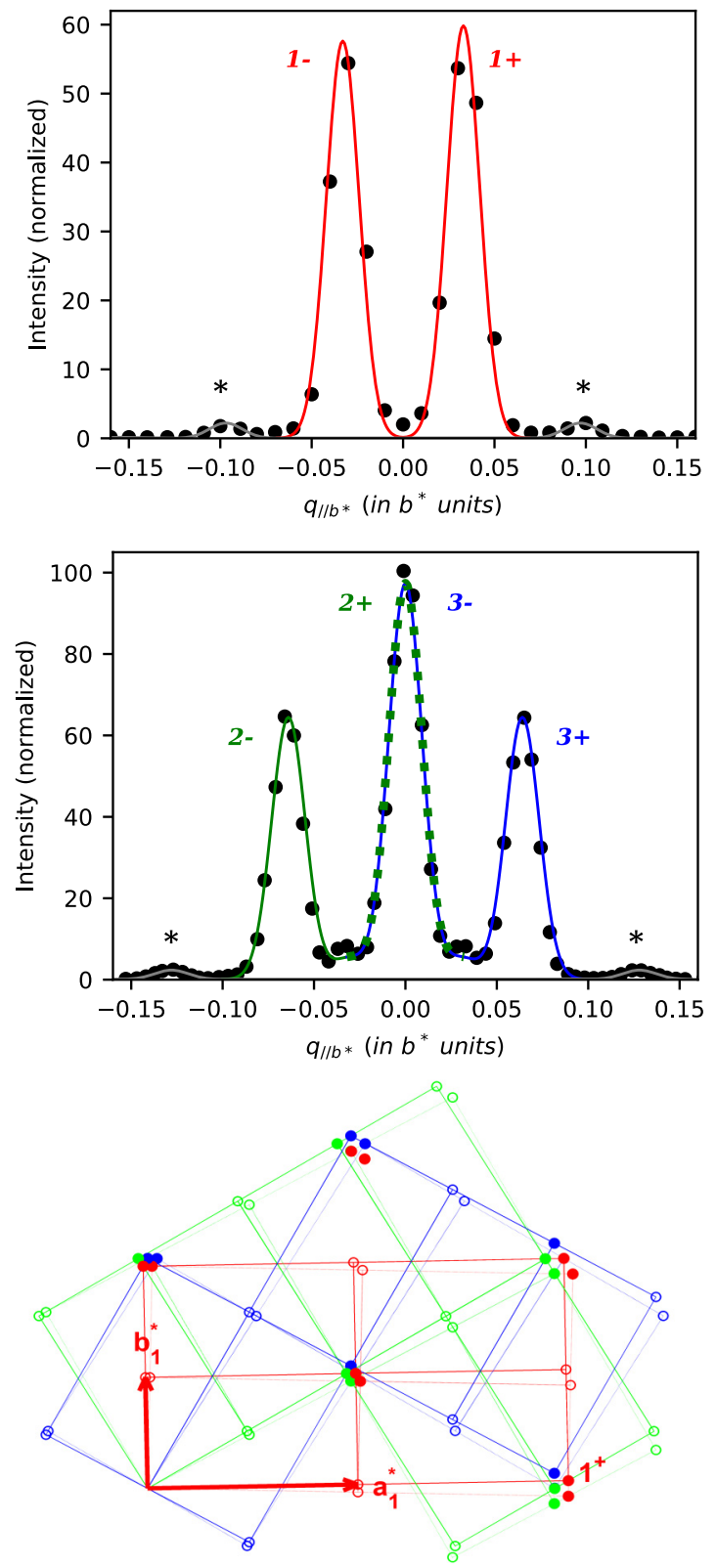

FIG. 1. Thermal neutron diffraction measured at the Laboratoire Léon Brillouin on a triple-axis spectrometer (1T) of the $\left(\boldsymbol{a}^{*}, \boldsymbol{b}^{*}\right)$ plane of $n$-nonadecane/urea in the ferroelastic phase. The measurement was performed at $T=100 \mathrm{~K}$ on a fully deuterated crystal. These two scans correspond to two measurements of the maxima of the intensity associated to the low-symmetry-induced domains. They are measured for $h=4$ and $k=0$, as defined in the schematic figure below considering the domain 1 . Top: measurement along $\boldsymbol{b}^{*}$ in $h=4$. Middle: same measurement in $h=3.86$. Bottom: schematic representation of the diffraction image, identifying the six different domains. Red, blue, and green refer to three types of symmetryinduced domains generated by the loss of the threefold symmetry element going from the hexagonal to the orthorhombic phase (domains 1,2, and 3, respectively). A supplementary splitting is observed with a small tilt angle apart from the mean value [24], defining six perfectly separate types of domains, respectively called $1+, 1-, 2+, 2-, 3+, 3-$. Further studies presented in this paper are performed in a single type of domain, conventionally called $1+$. The asterisk marks other small supplementary misoriented domains [40].
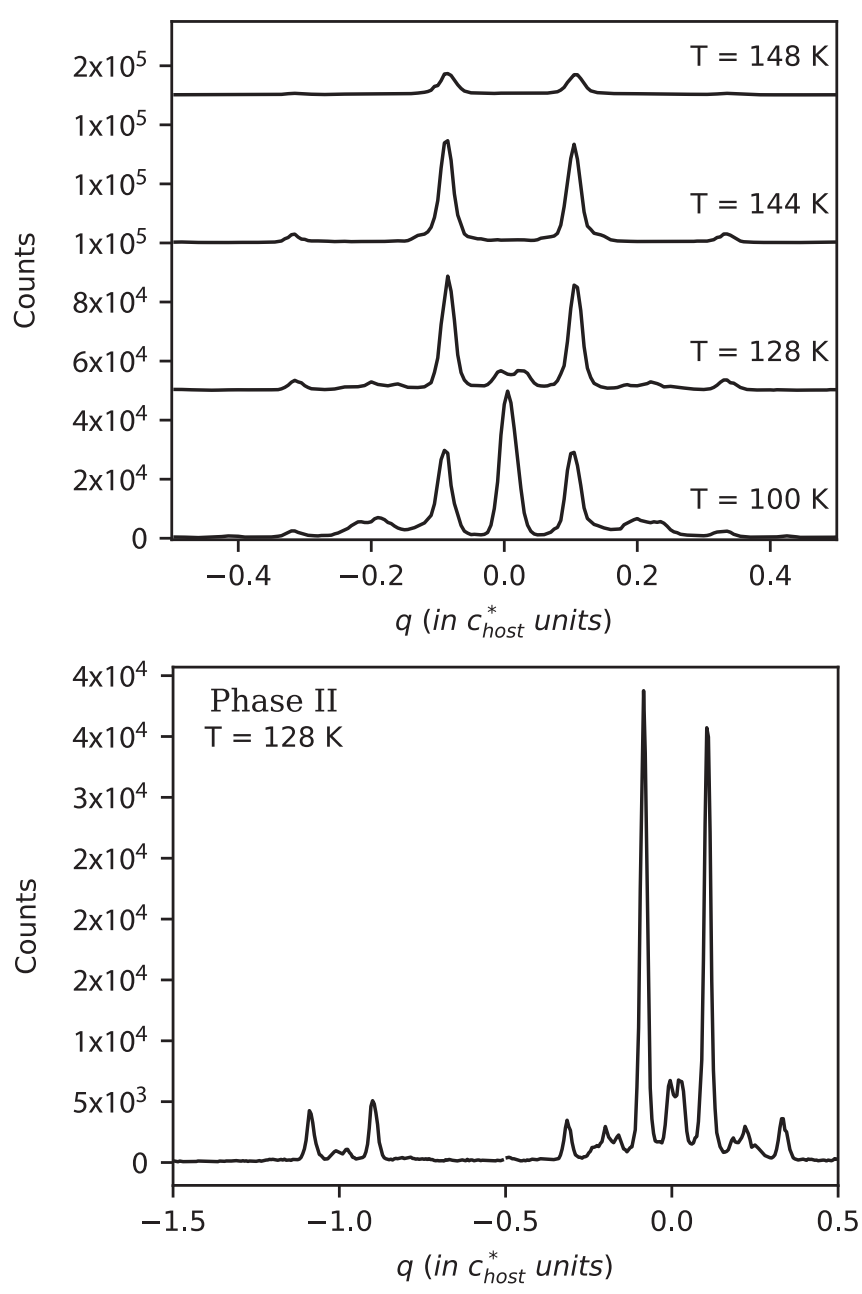

FIG. 2. Cold neutron diffraction measured at the Laboratoire Léon Brillouin on a triple-axis spectrometer $(4 \mathrm{~F})$. The sample was a fully deuterated $n$-nonadecane/urea single crystal $\left(T_{c 1}=149.4 \mathrm{~K}\right.$, $T_{c 2}=127.8 \mathrm{~K}$ ). Top: Limited $q$-range scan around common reciprocal position ( 3200 ), along $c^{*}$ from -0.5 to +0.5 in urea $c_{h}^{*}$ reciprocal units. Temperature decreasing from top to bottom, respectively, $T=148,144,128$, and $100 \mathrm{~K}$. Each curve is shifted by a value of $5 \times 10^{4}$ counts for clarity. Bottom: At $T=128 \mathrm{~K}$, full range scan along the same reciprocal $\boldsymbol{c}^{*}$ line around both the urea (3 $2-10)$ and common ( 3200 ) reciprocal position from -1.5 to +0.5 in urea $c_{h}^{*}$ reciprocal units.

According to this measurement, the wavelength of the supplementary modulation was of the order of $c_{h} / \delta=120 \AA$, where $\delta$ is found equal to $0.091 \pm 0.005$. A lower-ordered phase, called III, appears below $T_{c 2}^{D}=127.8 \mathrm{~K}$ in the fully deuterated $n$-nonadecane/urea. There, superstructure Bragg peaks are present in the common $l=0$ position, as shown at $T=100 \mathrm{~K}$ in Fig. 2 (top). The so-called satellites at $\pm \delta c_{h}^{*}$ are still present at this temperature, showing the persistence of the associated supplementary intermodulation. The lowest-temperature measurement by neutron scattering in phase II at $T=128 \mathrm{~K}$ reveals supplementary satellites very close to the common and the host superstructure position, but clearly still out of these positions, as shown in Fig. 2 (bottom).

In order to analyze these diffraction peaks, an even better spatial resolution is required. Therefore, high spatial 
resolution studies have been performed using the inelastic backscattering spectrometer ID28 at the synchrotron ESRF at Grenoble, France. The optical layout is based on the triple-axis principle. The energy resolution is obtained by a monochromator $\mathrm{Si}(12,12,12)$, in backscattering geometry. The scattered photons are energy analyzed by a perfect spherical silicon crystal analyzer, operated in Rowland geometry. The $\mathrm{x}$ rays diffracted from the analyzer crystal are recorded by solid-state detectors (low-noise silicon detectors), which present a very low background. The incident photon wavelength was then $\lambda=0.5227 \AA(E=23.7 \mathrm{keV})$ and the energy resolution was $1.5 \mathrm{meV}$ [39] in a wide part of the reciprocal space up to $10 \AA^{-1}$. This energy resolution means that only very low-frequency acoustic phonons are integrated within the presented elastic measurements. Such thermal diffuse scattering can thus be ignored. The spatial resolution is at least of the order of $0.7 \mu \mathrm{m}$, as discussed later on. The organic nature of the $n$-nonadecane/urea $\mathrm{C}_{19} \mathrm{H}_{40} / \mathrm{CO}\left(\mathrm{NH}_{2}\right)_{2}$ sample requires a large single crystal, in this case $0.5 \times 0.5 \times 1 \mathrm{~cm}^{3}$, in order to maximize the scattered intensity. The size of the crystals used for neutrons and inelastic x-ray scattering is similar and their growing is done according to the same process. This instrument is used as a triple-axis spectrometer, allowing the required scan within the reciprocal space. These studies were performed on a fully hydrogenated $\mathrm{C}_{19} \mathrm{H}_{40} / \mathrm{CO}\left(\mathrm{NH}_{2}\right)_{2}$ single crystal. Like in the neutron study, the diffraction measurements were performed on a single selected domain equivalent to the so-called domain $1+$. The use of a highly selective $\mathrm{x}$-ray backscattering monochromator drastically decreases the incoming x-ray flux, thus ensuring that no substantial damage is created in the samples during these data acquisitions.

\section{A. Critical pretransitional phenomena within the high-symmetry phase $P 6_{1} 22(00 \gamma)$}

Measurements were performed in the high-symmetry phase along the aperiodic direction $\boldsymbol{c}^{*}$, around the reciprocal $\left(\begin{array}{llll}3 & 0 & 3 & 0\end{array}\right)$ position. They reveal the existence of diffuse scattering with maxima around $\pm \delta c_{h}^{*}$ (see profiles shown in Fig. 3). Quite long correlation lengths $\xi$ are extracted from the fit of the data at $T=165 \mathrm{~K}$ by the usual $S(q, T)=$ $k_{B} T /\left(q^{2}+\xi^{-2}\right)$ function $: \xi=700 \AA$ at $T=175 \mathrm{~K}$ and $\xi=850 \AA$ at $T=165 \mathrm{~K}$. The feature of this critical diffuse scattering has been extensively discussed in [35]. The gray curves in Fig. 3 correspond to the diffraction profile of the Bragg peaks in the ordered phase II $(T=158 \mathrm{~K})$. According to the width of these peaks, the length of the long-range order within this crystal is of the order of $0.70 \mu \mathrm{m}$. The spatial resolution by neutron scattering is certainly defined by the spectrometer itself, whereas the x-ray backscattering spectrometer permits one to measure some Bragg broadening. The related typical peak width ( $1 \mathrm{mrad}$ FWHM) is superior to the beam divergence and is likely to be determined by the mosaicity of the crystal.

Such long correlation lengths within the high-symmetry phase reveal the very low-energy cost to generate spatial pretransitional fluctuations of the order parameter along the aperiodic direction [35]. In that direction, a specific excitation has been reported, the so-called sliding mode [48]. Its existence was revealed via a coupling with the longitudinal acoustic

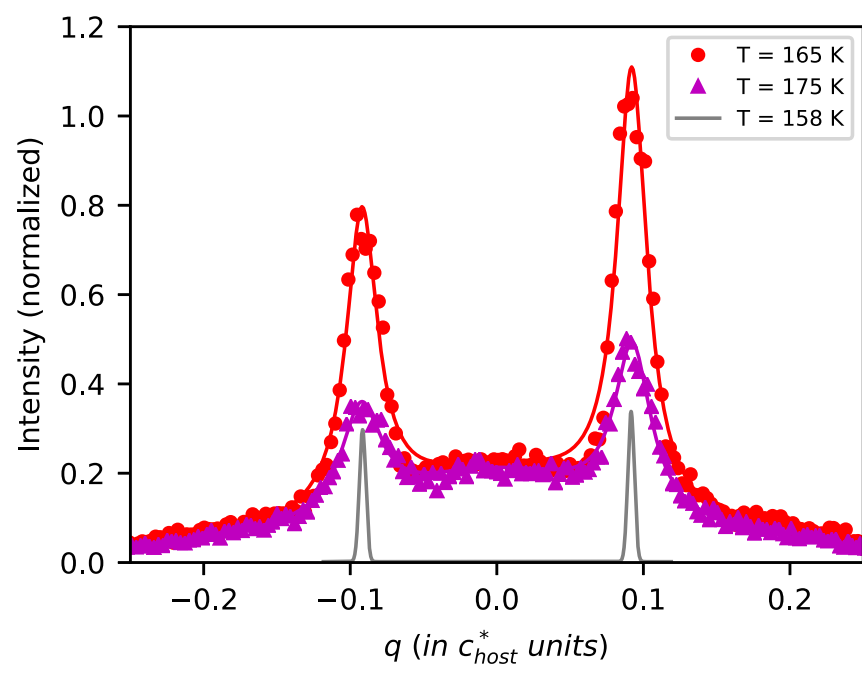

FIG. 3. Diffraction profiles measured on the inelastic x-ray backscattering spectrometer at the ESRF (ID28). Scan along the reciprocal direction $\boldsymbol{c}^{*}$ around the (3 030 ) host reciprocal position, above $T_{c 1}=160 \mathrm{~K}$ (fully hydrogenated $n$-nonadecane/urea single crystal), at $T=165 \mathrm{~K}$ (red circles) and $175 \mathrm{~K}$ (magenta triangles). The associated red (respectively magenta) solid lines are the refined profiles as described in the text. The same scan is shown in phase II ( $T=158 \mathrm{~K}$, gray solid line) to show the typical Bragg peak profile that defines the momentum resolution here.

phonon propagating along that direction. It corresponds in a theoretical description to the zero-energy antitranslational longitudinal mode of the host and guest subsystems. The softening of such an excitation may induce a supplementary intermodulation analog to a soft phonon mode which generates incommensurate static modulation in incommensurate modulated crystals [6]. Dynamical studies are in progress to test this hypothesis.

\section{B. The ordered ferroelastic phase II, between $T_{c 1}$ and $T_{c 2}$ : $C 222_{1}(00 \gamma)(10 \delta)$}

The measurement performed by neutron scattering in phase II at $T=128 \mathrm{~K}$ is shown in Fig. 2 (bottom) both around the host $(l=-1)$ and the common $(l=0)$ reciprocal point. Whereas some complexity appears in the scan, the intensity presents a minimum at these two positions. Actually, this measure reveals the already reported Bragg peaks at $\pm 0.091 \boldsymbol{c}_{h}^{*}$, but, in addition, other Bragg peaks much closer to the common and the host reciprocal positions. Figure 4 presents the measurement performed by inelastic synchrotron scattering of the fully hydrogenated single crystal in phase II at $T=135 \mathrm{~K}$. We could not measure the main Bragg peaks together as it would damage the detectors, but they were aligned with the absorber, and the scans along the reciprocal direction $\boldsymbol{c}^{*}$ were performed within a well-defined orientation matrix. For this reason, in order not to saturate the detector, the measurement could not be performed around the common reciprocal position, so only the study around the host reciprocal positions could be done. However, the neutron study shown in Fig. 2 (bottom) reveals that a similar behavior is reported around both host and common reciprocal positions. A very 


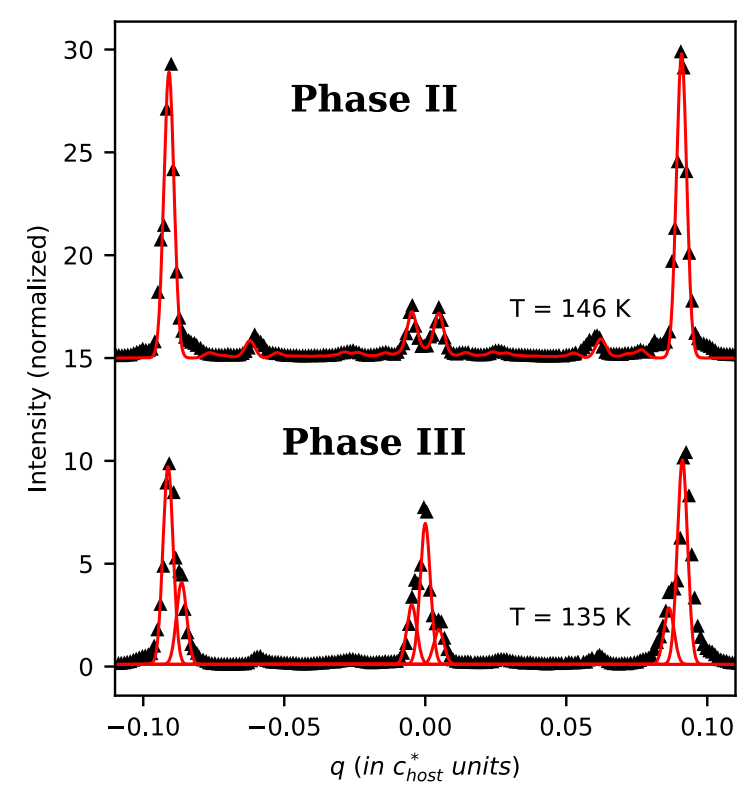

FIG. 4. Diffraction profiles measured on the inelastic x-ray backscattering spectrometer at the ESRF (ID28). Scan along the reciprocal direction $\boldsymbol{c}^{*}$ around the (3 030 ) host reciprocal position in phase II, $T=146 \mathrm{~K}$, and phase III, $T=135 \mathrm{~K}$. Measurements were performed on fully hydrogenated $n$-nonadecane/urea $\left(T_{c 1}=160 \mathrm{~K}\right.$, $T_{c 2}=145 \mathrm{~K}$ ). Measured data are shown with black triangles; refined patterns are shown with solid red lines. Here, the five-dimensional notation of the Bragg peaks is used : $\boldsymbol{Q}_{h k l m n}=\mathrm{h} \boldsymbol{a}^{*}+\mathrm{k} \boldsymbol{b}^{*}+1$ $\boldsymbol{c}_{h}^{*}+\mathrm{m} \boldsymbol{c}_{g}^{*}+\mathrm{n} \boldsymbol{c}_{m}^{*}$ (see text). In phase II, the fitted Bragg peaks fulfill the condition of the presence of $h+k+\mathrm{n}$ even, where the fifth supplementary parameter is $\boldsymbol{c}_{m}{ }^{*}=(0.0048 \pm 0.0005) \boldsymbol{c}_{h}{ }^{*}$. In phase II, we report significant satellite Bragg peaks with $\mathrm{n}= \pm 1$, $\pm 13, \pm 19$. For phase III, individual contributions of the refined Bragg peaks are also displayed. Bragg peaks appear with $\mathrm{n}=0$ and $\mathrm{n}= \pm 18$.

complex structure is reported by $\mathrm{x}$-ray synchrotron diffraction with a series of maxima as shown in Fig. 4. Note that in this measurement, horizontal beam divergence was equal to 150 $\mu \mathrm{rad}$ (FWHM), which is still an order of magnitude smaller than the splitting of satellites along $\mathrm{c}^{*}[ \pm 1 \mathrm{mrad}$, determined around the ( $\left.\begin{array}{lllll}3 & 0 & 3 & 0 & 0\end{array}\right)$ Bragg peak position]. The main point is that with this high spatial resolution, the intensity is zero in the host reciprocal position. The second major feature is the new value for the misfit parameter extracted from these data. According to the fit shown in Fig. 4, this value is found equal to $0.0048 \pm 0.0005$ in $c_{h}^{*}$ units. With such a very short reciprocal vector, values of the indices $l$ and $m$ higher than 100 would be needed to express the position of this satellite in the four-dimensional combination $\left(l c_{h}^{*}+m c_{g}^{*}\right)$ (within the precision of our data). So, such a description can be ruled out here. Following the previous assumption of a five-dimensional superspace characterized by five indices $(h, k, l, m, n)$, we define the following vector: $\boldsymbol{Q}_{h k l m n}=h \boldsymbol{a}^{*}+k \boldsymbol{b}^{*}+l \boldsymbol{c}_{h}^{*}+m \boldsymbol{c}_{g}^{*}+n \boldsymbol{c}_{m}^{*}$, where $\boldsymbol{c}_{m}^{*}=\delta \boldsymbol{c}_{h}^{*}$. In Fig. 4 , the satellites are indexed by only odd numbers $(n=1,3, \ldots)$. The strong maxima at \pm 0.091 corresponds to $n=19$ considering this new reciprocal periodicity. The wavelength of this intermodulation is given by $c_{m}=c_{h} / \delta$ : this reciprocal position corresponds to a value of the wavelength of the supplementary incommensurate intermodulation along the channels of the order of $0.23 \mu \mathrm{m}$, which is extremely large. Due to the sensibility of the detector, the high resolution of this spectrometer, and the exceptional mosaicity of the crystal, this very long period of the supplementary modulation can be observed by inelastic $x$-ray scattering.

The systematic condition of the absence of the host and common superstructure $h+k+n$ odd observed in phase II is in favor of the five-dimensional superspace number 20.2.24.2 [49]: $C 222_{1}(00 \gamma)(10 \delta)$. This superspace group is a subgroup of the high-symmetry superspace group $P 6_{1} 22(00 \gamma)$, considering a critical wave vector $\left(\begin{array}{lllll}1 / 2 & 1 / 2 & 0 & 0 & 1 / 2\end{array}\right)$ within this high-temperature phase. The scientific meaning of this phase group as applied to $n$-nonadecane/urea is explicitly given in [35]. The fifth parameter is associated to the appearance of a supplementary intermodulation of both host and guest sublattices along the channel direction. This intermodulation is in antiphase for adjacent channels along the diagonal of the orthorhombic phase (see Fig. 2 in Ref. [35]). Unfortunately, it is not possible to perform a structure refinement in the low-temperature phases because there are so many symmetryinduced domains, as shown in Fig. 1.

\section{The ordered phase III, between $T_{c 2}$ and $T_{c 3}$ : $P 22_{1} 2_{1}(00 \gamma)(00 \delta)$}

An essential change appears in the temperature range between $T=146$ and $T=135 \mathrm{~K}$, as shown in the diffraction patterns in Fig. 4 (bottom). We observe a new phase, called phase III, signed by the appearance of very narrow superstructure Bragg peaks here in the host reciprocal position. This symmetry breaking is more clearly illustrated in Fig. 5 with a zoom on the central part of the diffraction image around the host reciprocal position. In the neutron-scattering measurements, a similar appearance of a superstructure Bragg peak at common reciprocal position is observed as the signature of phase III (Fig. 2). This result confirms that the structural change around $T_{c 2}=145 \mathrm{~K}$ is a real phase transition, associated to the loss of the centering within a five-dimensional space. No condition of absence exists any longer for $h+k+n$ in this phase III, where the C centering is lost, leading to a primitive unit cell in phase III. This phase is described by a five-dimensional superspace group number 19.2.18.1 [49] $P 2{ }_{1} 2_{1} 2_{1}(00 \gamma)(00 \delta)$, which is a subgroup of the superspace group of phase II. Let us mention that a supplementary phase appears below a temperature $T_{c 3}$ of the order of $60 \mathrm{~K}$ in the fully deuterated sample [37].

\section{CONCLUSION}

Superspace crystallography is the tool to describe aperiodic crystals, recovering translational symmetry within higher-dimensional space larger than three. The $n$-alkane/urea compounds are prototype examples of incommensurate composite crystals. Most of them are described at room temperature by a four-dimensional hexagonal superspace group. From the first work [22] and over more than three decades [23-27], the aperiodic feature was not considered there explicitly and a three-dimensional (3D) description was given when consid- 

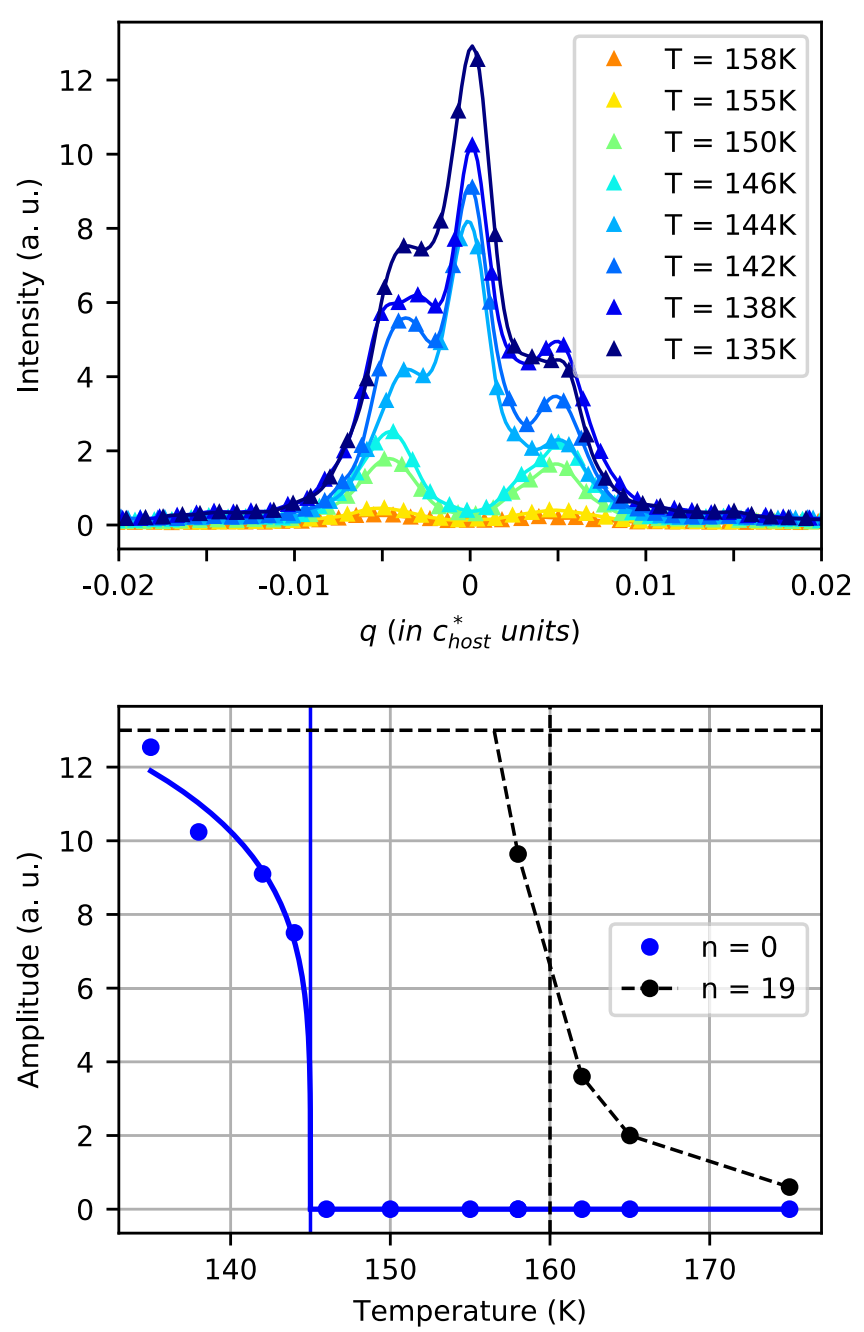

FIG. 5. Diffraction profiles measured on the inelastic x-ray backscattering spectrometer at the ESRF (ID28). Top: Zoom on the scan along the reciprocal direction $\boldsymbol{c}^{*}$ around the $\left(\begin{array}{llll}3 & 0 & 3 & 0\end{array}\right)$ host reciprocal position. Fully hydrogenated $n$-nonadecane/urea $\left(T_{c 1}=160 \mathrm{~K}, T_{c 2}=145 \mathrm{~K}\right)$ in phase II and phase III. Bottom: Integrated intensity at satellite position ( $\left.\begin{array}{lllll}3 & 0 & 3 & 0 & 19\end{array}\right)$ verifying the condition of the presence of $h+k+n$ even (black) and position

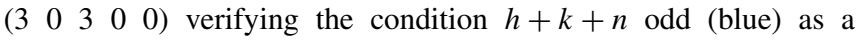
function of temperature, defining the clear sequence of the three phases.

ering the phase transition. We had later shown that the phase transition requires a $5 \mathrm{D}$ description for the low-temperature phases of $n$-nonadecane/urea [33]. There is a fundamental structural change when considering the previous 3D description (which is actually a bidimensional description) and the 5D one. In a theoretical article, Lynden-Bell [26] considered one single-phase transition for all $n$-alkane/urea, describing the ordering in the commensurate basal plane $(\boldsymbol{a}, \boldsymbol{b})$, perpendicular to the channels. With her calculation, she found two possible structural solutions for this phase transition. One is an antiferroshearing of the host urea sublattice with a herringbone ordering of the guest: this induces a doubling in the basal plane perpendicular to the incommensurate channel axis. At that time, due to bad spatial resolution, the superstructure Bragg peaks were indeed observed by error in the basal reciprocal space $\left(\boldsymbol{a}^{*}, \boldsymbol{b}^{*}\right)$ in $h+k$ odd positions. So, the community agreed on a single-phase transition from hexagonal $P 6_{1} 22$ to orthorhombic $P 2_{1} 2_{1} 2_{1}$ [23-27]. The second solution corresponds to an ordering of the alkane molecules that are all parallel and a ferroshearing of the host. This meant no Bragg peaks in the $\left(\boldsymbol{a}^{*}, \boldsymbol{b}^{*}\right)$ plane at $h+k$ odd, as we indeed report in phase II. Of course, this is a quite limited representation of the structural ordering within this phase. In addition, since satellite Bragg peaks appear along the incommensurate directions out of the $\left(\boldsymbol{a}^{*}, \boldsymbol{b}^{*}\right)$ plane at $h+k$ odd, we have to assume a doubling of the unit cell within the crystallographic superspace. This C-centered $C 222_{1}(00 \gamma)(10 \delta)$ five-dimensional superspace group for phase II has been extensively described in previous articles $[36,38]$. The present study reports in $n$-nonadecane/urea a wavelength for the new incommensurate intermodulation along the aperiodic direction $(0.23 \mu \mathrm{m})$ that is much longer than the one previously reported doing neutron-scattering studies $[31,33]$ due to the very high-momentum resolution of the $\mathrm{x}$-ray backscattering spectrometer. This very high resolution also permits a clear determination of the temperature evolution of the order parameter signing the second symmetry breaking at $T_{c 2}$ leading to the superspace group $P 2_{1} 2_{1} 2_{1}(00 \gamma)(00 \delta)$. The same very long wavelength of the supplementary modulation $(0.23 \mu \mathrm{m})$ is found in this phase III according to the precision of our data. As mentioned in Sec. I, recently two studies argued that there is one single-phase transition in $n$-nonadecane/urea from $P 6_{1} 22(00 \gamma)$ to $P 2_{1} 2_{1} 2_{1}(00 \gamma)$ [42-44], as initially proposed. Their model considers again the critical point $M$ within the reciprocal $\left(\boldsymbol{a}^{*}, \boldsymbol{b}^{*}\right)$ plane [44]. We have shown here that this is not correct: in phase II, the critical wave vector is outside the $\left(\boldsymbol{a}^{*}, \boldsymbol{b}^{*}\right)$, in the point $(1 / 2,1 / 2,0,0,1 / 2)$ of the five-dimensional Brillouin zone $[33,36,38]$. It corresponds to a completely different symmetry within a Landau description of the phase transition. This work shows that incommensurate composite crystals may present complicated structural features which can only be revealed using very high-momentum resolution diffractometers. The inelastic x-ray backscattering spectrometer used here fits these requirements. This generation of instruments gives new opportunities to study symmetry breakings and their related critical pretransitional phenomena with new perspectives. This opens a large field of research for revisiting phase transitions in matter.

\section{ACKNOWLEDGMENTS}

We thank T. Breczewski for providing the large fully hydrogenated and fully deuterated single crystals, and M. D. Hollingsworth for very fruitful discussions. M.V. acknowledges the support from SNSF Grant No. 200021L_169753 and European Union's Horizon 2020 research and innovation program under the Marie Skłodowska-Curie Grant No. 701647. 
[1] L. Landau and E. Lifshitz, Statistical Physics, 3rd ed. (Butterworth-Heinemann, Oxford, 1980).

[2] K. G. Wilson, Rev. Mod. Phys. 55, 583 (1983).

[3] P. M. de Wolff, T. Janssen, and A. Janner, Acta Crystallogr. Sect. A 37, 625 (1981).

[4] T. Janssen, A. Janner, A. Looijenga-Vos, and P. M. de Wolff, in International Tables for Crystallography, edited by H. Fuess, T. Hahn, H. Wondratschek, U. Müller, U. Shmueli, E. Prince, A. Authier, V. Kopský, D. B. Litvin, M. G. Rossmann, E. Arnold, S. R. Hall, B. McMahon, and E. Prince (International Union of Crystallography, Chester, UK, 2006), pp. 907-955.

[5] S. van Smaalen, Incommensurate Crystallography (Oxford University Press, Oxford, 2007).

[6] R. R. Blinc and A. P. Levanyuk, Incommensurate Phases in Dielectrics (North-Holland, Amsterdam, 1986).

[7] T. Janssen, G. Chapuis, and M. de Boissieu, Aperiodic Crystals : From Modulated Phases to Quasicrystals: Structure and Properties (Oxford University Press, Oxford, 2007).

[8] P. Coppens, Acta Crystallogr. Sect. B 51, 402 (1995).

[9] S. Van Smaalen and K. D. M. Harris, Proc. R. Soc. London A 452, 677 (1996).

[10] D. Shechtman, I. Blech, D. Gratias, and J. W. Cahn, Phys. Rev. Lett. 53, 1951 (1984).

[11] D. Gratias and M. Quiquandon, Philos. Mag. 88, 1887 (2008).

[12] T. Janssen, Acta Crystallogr. Sect. A 68, 667 (2012).

[13] J. C. Tolédano and P. Tolédano, The Landau Theory of Phase Transitions (World Scientific, Singapore, 1987).

[14] H. T. Stokes and B. J. Campbell, Acta Crystallogr. Sect. A 73, 4 (2017).

[15] P. A. Albouy, J. P. Pouget, and H. Strzelecka, Phys. Rev. B 35, 173 (1987).

[16] J. M. Hastings, J. P. Pouget, G. Shirane, A. J. Heeger, N. D. Miro, and A. G. MacDiarmid, Phys. Rev. Lett. 39, 1484 (1977).

[17] I. U. Heilmann, J. D. Axe, J. M. Hastings, G. Shirane, A. J. Heeger, and A. G. MacDiarmid, Phys. Rev. B 20, 751 (1979).

[18] M. I. McMahon and R. J. Nelmes, Phys. Rev. Lett. 93, 055501 (2004).

[19] E. Castillo-Martínez, A. Schönleber, S. van Smaalen, A. Arévalo-López, and M. Alario-Franco, J. Solid State Chem. 181, 1840 (2008).

[20] R. C. Pemberton and N. G. Parsonage, Trans. Faraday Soc. 61, 2112 (1965).

[21] H.-U. Lenné, H.-C. Mez, and W. Schlenk Jr., J. Liebigs Annal. Chem. 732, 70 (1970).

[22] Y. Chatani, H. Anraku, and Y. Taki, Mol. Cryst. Liq. Cryst. 48, 219 (1978).

[23] R. Forst, H. Boysen, F. Frey, H. Jagodzinski, and C. Zeyen, J. Phys. Chem. Solids 47, 1089 (1986).

[24] R. Forst, H. Jagodzinski, H. Boysen, and F. Frey, Acta Crystallogr. Sect. B 46, 70 (1990).

[25] K. D. M. Harris and J. M. Thomas, J. Chem. Soc., Faraday Trans. 86, 2985 (1990).

[26] R. Lynden-Bell, Molec. Phys. 79, 313 (1993).

[27] M. D. Hollingsworth and K. D. M. Harris, Urea, thiourea and selenourea inclusion compounds, in Comprehensive Supramolecular Chemistry, edited by D. D. MacNicol (Elsevier Science, Oxford, 1996), Vol. 6, Chap. 7, pp. 177-237.

[28] T. Weber, H. Boysen, M. Honal, F. Frey, and R. B. Neder, Z. Kristallogr. Crystal. Mater. 211, 238 (1996).
[29] R. Lefort, J. Etrillard, B. Toudic, F. Guillaume, T. Breczewski, and P. Bourges, Phys. Rev. Lett. 77, 4027 (1996).

[30] T. Weber, H. Boysen, and F. Frey, Acta Crystallogr. Sect. B 56, 132 (2000).

[31] B. Toudic, P. Garcia, C. Odin, P. Rabiller, C. Ecolivet, E. Collet, P. Bourges, G. J. McIntyre, M. D. Hollingsworth, and T. Breczewski, Science 319, 69 (2008).

[32] B. Toudic, F. Aubert, C. Ecolivet, P. Bourges, and T. Breczewski, Phys. Rev. Lett. 96, 145503 (2006).

[33] B. Toudic, P. Rabiller, L. Bourgeois, M. Huard, C. Ecolivet, G. J. McIntyre, P. Bourges, T. Breczewski, and T. Janssen, Europhys. Lett. 93, 16003 (2011).

[34] C. Mariette, M. Huard, P. Rabiller, S. M. Nichols, C. Ecolivet, T. Janssen, K. E. Alquist, M. D. Hollingsworth, and B. Toudic, J. Chem. Phys. 136, 104507 (2012).

[35] C. Mariette, L. Guérin, P. Rabiller, C. Ecolivet, P. GarcíaOrduña, P. Bourges, A. Bosak, D. de Sanctis, M. D. Hollingsworth, T. Janssen, and B. Toudic, Phys. Rev. B 87, 104101 (2013).

[36] L. Guérin, C. Mariette, P. Rabiller, M. Huard, S. Ravy, P. Fertey, S. M. Nichols, B. Wang, S. C. B. Mannsfeld, T. Weber, M. D. Hollingsworth, and B. Toudic, Phys. Rev. B 91, 184101 (2015).

[37] S. Zerdane, C. Mariette, G. J. McIntyre, M.-H. Lemée-Cailleau, P. Rabiller, L. Guérin, J. C. Ameline, and B. Toudic, Acta Crystallogr. Sect. B 71, 293 (2015).

[38] C. Mariette, I. Frantsuzov, B. Wang, L. Guérin, P. Rabiller, M. D. Hollingsworth, and B. Toudic, Phys. Rev. B 94, 184105 (2016).

[39] C. Ecolivet, M. Verezhak, C. Mariette, L. Guérin, P. Rabiller, J. Ollivier, A. Bosak, and B. Toudic, Phys. Rev. B 98, 224308 (2018).

[40] T. Weber, Modulated Structures and disorder in Urea inclusion compounds as a function of temperature from $30 \mathrm{~K}$ to 300 K, Ph.D. Thesis, Ludwig-Maximilians-Universität München, 1997.

[41] A. López-Echarri, I. Ruiz-Larrea, A. Fraile-Rodríguez, J. DíazHernández, T. Breczewski, and E. H. Bocanegra, J. Phys. Condens. Matter 19, 186221 (2007).

[42] M. Couzi, F. Guillaume, K. D. M. Harris, B. A. Palmer, K. Christensen, and S. P. Collins, Europhys. Lett. 116, 56001 (2016).

[43] K. Christensen, P. A. Williams, R. Patterson, B. A. Palmer, M. Couzi, F. Guillaume, and K. D. M. Harris, R. Soc. Open Sci. 6, 190518 (2019).

[44] M. Couzi, F. Guillaume, and K. D. M. Harris, R. Soc. Open Sci. 5, 180058 (2018).

[45] B. Toudic, L. Guérin, C. Mariette, I. Frantsuzov, P. Rabiller, C. Ecolivet, T. Janssen, and M. D. Hollingsworth, Europhys. Lett. 119, 66004 (2017).

[46] B. Toudic, L. Guérin, C. Mariette, I. Frantsuzov, P. Rabiller, C. Ecolivet, and M. D. Hollingsworth, R. Soc. Open Sci. 6, 182073 (2019).

[47] T. Weber, H. Boysen, F. Frey, and R. B. Neder, Acta Crystallogr. Sect. B 53, 544 (1997).

[48] B. Toudic, R. Lefort, C. Ecolivet, L. Guérin, R. Currat, P. Bourges, and T. Breczewski, Phys. Rev. Lett. 107, 205502 (2011).

[49] H. T. Stokes, B. J. Campbell, and S. van Smaalen, Acta Crystallogr. Sect. A 67, 45 (2011). 